\title{
TORSION UNITS IN INTEGRAL GROUP RING OF THE MATHIEU SIMPLE GROUP $M_{22}$
}

\author{
V. A. BOVDI, A. B. KONOVALOV AND S. LINTON
}

\begin{abstract}
We investigate the possible character values of torsion units of the normalized unit group of the integral group ring of the Mathieu sporadic group $M_{22}$. We confirm the Kimmerle conjecture on prime graphs for this group and specify the partial augmentations for possible counterexamples to the stronger Zassenhaus conjecture.
\end{abstract}

\section{Introduction, conjectures and main results}

Let $V(\mathbb{Z} G)$ be the normalized unit group of the integral group ring $\mathbb{Z} G$ of a finite group $G$. A long-standing conjecture of H. Zassenhaus (ZC) says that every torsion unit $u \in V(\mathbb{Z} G)$ is conjugate within the rational group algebra $\mathbb{Q} G$ to an element in $G$ (see $[\mathbf{2 5}])$.

For finite simple groups the main tool for the investigation of the Zassenhaus conjecture is the Luthar-Passi method, introduced in [20] for the case of $A_{5}$ and then applied in [21] for the case of $S_{5}$. Later M. Hertweck in [15] extended the method and applied it to $\operatorname{PSL}\left(2, p^{n}\right)$. The same method has also proved to be useful for some groups containing non-trivial normal subgroups. For some recent results we refer to $[\mathbf{5}, \mathbf{7}, \mathbf{1 4}, \mathbf{1 6}, \mathbf{1 5}, \mathbf{1 7}]$. Some related properties and weakened variations of the Zassenhaus conjecture can be found in $[\mathbf{1}, \mathbf{3}, \mathbf{1 9}]$.

To define the conjectures we will investigate, and describe the methods we will use, we introduce some notation. By $\#(G)$ we denote the set of all primes dividing the order of $G$. The Gruenberg-Kegel graph (or the prime graph) of $G$ is the graph $\pi(G)$ with vertices labeled by $\#(G)$ and an edge from $p$ to $q$ (for $p \neq q$ ) if there is an element of order $p q$ in $G$. In [19], W. Kimmerle proposed the following weakened variation of the Zassenhaus conjecture:

(KC) If $G$ is a finite group then $\pi(G)=\pi(V(\mathbb{Z} G))$.

It is easy to see that (ZC) implies $\mathbf{K C}$ since it implies that the set of orders of torsion units of $V(\mathbb{Z} G)$ is the same the set of orders of elements of $G$.

In $([\mathbf{1 9}], \S 4)$ it is shown that $(\mathbf{K C})$ holds for finite Frobenius and solvable groups. We remark that with respect to the so-called $p$-version of the Zassenhaus conjecture the investigation of Frobenius groups was completed by M. Hertweck and the first author in $[\mathbf{4}]$. In $[\mathbf{6}, \mathbf{7}, \mathbf{8}, \mathbf{1 0}]$, (KC) was confirmed for the sporadic simple groups $M_{11}, M_{12}, M_{23}$ and some Janko simple groups.

The research was supported by OTKA grants no. T037202, no. T038059, no. K68383 and Francqui Stichting (Belgium) grant ADSI107

Received 24 April 2007, revised 25 September 2007; published 8 February 2008.

2000 Mathematics Subject Classification 16S34, 20C05, 20D08

(C) 2008, V. A. Bovdi, A. B. Konovalov and S. Linton 
Here we continue these investigations for the Mathieu simple group $M_{22}$. Although we cannot prove the rational conjugacy of torsion units of $V\left(\mathbb{Z} M_{22}\right)$ with elements of $M_{22}$, our main result gives a lot of information on the orders and partial augmentations of these units. In particular, we confirm Kimmerle's conjecture for this group.

Let $G=M_{22}$. It is well known (see $[\mathbf{1 3}, \mathbf{2 4}]$ ) that $|G|=2^{7} \cdot 3^{2} \cdot 5 \cdot 7 \cdot 11$ and $\exp (G)=2^{3} \cdot 3 \cdot 5 \cdot 7 \cdot 11$. The group $G$ has 12 irreducible characters of the following degrees: 1, 21, 45, 45, 55, 99, 154, 210, 231, 280, 280 and 385. Let

$$
\mathcal{C}=\left\{C_{1}, C_{2 a}, C_{3 a}, C_{4 a}, C_{4 b}, C_{5 a}, C_{6 a}, C_{7 a}, C_{7 b}, C_{8 a}, C_{11 a}, C_{11 b}\right\}
$$

be the collection of all conjugacy classes of $M_{22}$, where the first index denotes the order of the elements of this conjugacy class and $C_{1}=\{1\}$. Suppose $u=\sum \alpha_{g} g \in$ $V(\mathbb{Z} G)$ has finite order $k>1$. Denote by $\nu_{n t}=\nu_{n t}(u)=\varepsilon_{C_{n t}}(u)=\sum_{g \in C_{n t}} \alpha_{g}$ the partial augmentation of $u$ with respect to $C_{n t}$. From the Berman-Higman Theorem (see [2] and [23], Ch. 5, p. 102) one knows that $\nu_{1}=\alpha_{1}=0$ and

$$
\sum_{C_{n t} \in \mathcal{C}} \nu_{n t}=1 .
$$

Hence, for any character $\chi$ of $G$, we get that $\chi(u)=\sum \nu_{n t} \chi\left(h_{n t}\right)$, where $h_{n t}$ is a representative of the conjugacy class $C_{n t}$.

Our main result is the following theorem.

TheOREM 1. Let $G$ denote the Mathieu simple group $M_{22}$. Let $u$ be a torsion unit of $V(\mathbb{Z} G)$ of order $|u|$. Denote by $\mathfrak{P}(u)$ the tuple

$$
\left(\nu_{2 a}, \nu_{3 a}, \nu_{4 a}, \nu_{4 b}, \nu_{5 a}, \nu_{6 a}, \nu_{7 a}, \nu_{7 b}, \nu_{8 a}, \nu_{11 a}, \nu_{11 b}\right) \in \mathbb{Z}^{11}
$$

of partial augmentations of $u$ in $V(\mathbb{Z} G)$. The following properties hold.

(i) There are no elements of order $10,14,15,21,22,33,35,55$ or 77 in $V(\mathbb{Z} G)$. Equivalently, if $|u| \notin\{12,24\}$, then $|u|$ is the order of some element $g \in G$.

(ii) If $|u| \in\{2,3,5\}$, then $u$ is rationally conjugate to some $g \in G$.

(iii) If $|u|=4$, then all components of $\mathfrak{P}(u)$ are zero except possibly $\nu_{2 a}, \nu_{4 a}$ and $\nu_{4 b}$, and the triple $\left(\nu_{2 a}, \nu_{4 a}, \nu_{4 b}\right)$ is one of

$$
\begin{aligned}
& \{(-2,-1,4),(2,-1,0),(0,-1,2),(0,6,-5),(-2,6,-3),(0,5,-4) \text {, } \\
& (-2,5,-2),(-2,4,-1),(2,4,-5),(0,4,-3),(-2,3,0),(2,3,-4),(0,3,-2) \text {, } \\
& (2,0,-1),(-2,0,3),(0,0,1),(0,-6,7),(2,-6,5),(0,2,-1),(2,2,-3) \text {, } \\
& (-2,2,1),(0,-5,6),(2,-5,4),(0,-4,5),(2,-4,3),(-2,-3,6),(0,-3,4) \text {, } \\
& (2,-3,2),(-2,-2,5),(0,-2,3),(2,-2,1),(0,1,0),(-2,1,2),(2,1,-2)\} \text {. }
\end{aligned}
$$

(iv) If $|u|=6$, then all components of $\mathfrak{P}(u)$ are zero except possibly $\nu_{2 a}, \nu_{3 a}$ and $\nu_{6 a}$, and the triple $\left(\nu_{2 a}, \nu_{3 a}, \nu_{6 a}\right)$ is one of

$$
\begin{aligned}
& \{(-4,6,-1),(-2,6,-3),(4,-9,6),(-4,9,-4),(-2,3,0), \\
& \quad(-4,3,2),(0,3,-2),(2,0,-1),(-2,0,3),(0,0,1), \\
& (2,-6,5),(4,-6,3),(0,-3,4),(4,-3,0),(2,-3,2)\} .
\end{aligned}
$$

(v) If $|u|=7$, then all components of $\mathfrak{P}(u)$ are zero except possibly $\nu_{7 a}$ and $\nu_{7 b}$ 
and the pair $\left(\nu_{7 a}, \nu_{7 b}\right)$ is one of

$$
\{(0,1),(2,-1),(1,0),(-1,2)\} .
$$

(vi) If $|u|=11$, then all components of $\mathfrak{P}(u)$ are zero except possibly $\nu_{11 a}$ and $\nu_{11 b}$ and the pair $\left(\nu_{11 a}, \nu_{11 b}\right)$ is one of

$$
\begin{aligned}
& \{(5,-4),(0,1),(-2,3),(2,-1),(-3,4), \\
& \quad(-4,5),(1,0),(3,-2),(-1,2),(4,-3)\} .
\end{aligned}
$$

Note that using our implementation of the Luthar-Passi method (including Hertweck's extension), which we intend to make available in the GAP package LAGUNA [9], we are able to compute the set of 76 tuples containing (likely as a proper subset) possible tuples of partial augmentations for units of order 8, listed in Appendix 1. For the case of order 12 in Appendix 2 we listed 1166 tuples which can not be eliminated using the Luthar-Passi method.

As an immediate consequence of part (i) of the Theorem we obtain the corollary.

Corollary 1. If $G \cong M_{22}$ then $\pi(G)=\pi(V(\mathbb{Z} G))$.

\section{Preliminaries}

The following result allows a reformulation of the Zassenhaus conjecture in terms of vanishing of partial augmentations of torsion units.

Proposition 1 (see [20] and Theorem 2.5 in $[\mathbf{2 2}]$ ). Let $u \in V(\mathbb{Z} G$ ) be of order $k$. Then $u$ is conjugate in $\mathbb{Q} G$ to an element $g \in G$ if and only if for each $d$ dividing $k$ there is precisely one conjugacy class $C$ with partial augmentation $\varepsilon_{C}\left(u^{d}\right) \neq 0$.

The next results now serve to restrict the possible values of the partial augmentations of torsion units.

Proposition 2 (see [14], Proposition 3.1; [15], Proposition 2.2). Let $G$ be a finite group and let $u$ be a torsion unit in $V(\mathbb{Z} G)$. If $x$ is an element of $G$ whose p-part, for some prime $p$, has order strictly greater than the order of the p-part of $u$, then $\varepsilon_{x}(u)=0$.

The next result is explained in detail in $[\mathbf{2 0}]$ and $[\mathbf{4}, \mathbf{1 5}]$.

Proposition 3 (see $[\mathbf{1 5}, \mathbf{2 0}]$ ). Let either $p=0$ or $p$ a prime divisor of $|G|$. Suppose that $u \in V(\mathbb{Z} G)$ has finite order $k$ and assume $k$ and $p$ are coprime in case $p \neq 0$. If $z$ is a complex primitive $k$ th root of unity and $\chi$ is either a classical character or a $p$-Brauer character of $G$, then for every integer $l$ the number

$$
\mu_{l}(u, \chi, p)=\frac{1}{k} \sum_{d \mid k} \operatorname{Tr}_{\mathbb{Q}\left(z^{d}\right) / \mathbb{Q}}\left\{\chi\left(u^{d}\right) z^{-d l}\right\}
$$

is a non-negative integer.

Note that if $p=0$, we will use the notation $\mu_{l}(u, \chi, *)$ for $\mu_{l}(u, \chi, 0)$.

Finally, we shall use the well-known bound for orders of torsion units.

Proposition 4 (see [11]). The order of a torsion element $u \in V(\mathbb{Z} G)$ is a divisor of the exponent of $G$. 
In case of units of prime power order, the following Proposition may also be useful to eliminate some tuples of partial augmentations.

Proposition 5 (see [11]). Let $p$ be a prime, and let $u$ be a torsion unit of $V(\mathbb{Z} G)$ of order $p^{n}$. Then for $m \neq n$ the sum of all partial augmentations of $u$ with respect to conjugacy classes of elements of order $p^{m}$ is divisible by $p$.

\section{Proof of the Theorem}

Throughout this section we denote $M_{22}$ by $G$. The ordinary and $p$-Brauer character tables of $G$, which will be denoted by $\mathfrak{B C T}(p)$ where $p \in\{2,3,5,7,11\}$, can be found using the computational algebra system GAP [13], which derives its data from $[\mathbf{1 2}, \mathbf{1 8}]$. For the characters and conjugacy classes we will use throughout the paper the same notation, including indexation, as used in the GAP Character Table Library.

Since the group $G$ possesses elements of orders $2,3,4,5,6,7,8$ and 11, we first investigate units of all of these orders except 8. After this, since by Proposition 4 , the order of each torsion unit divides the exponent of $G$, it remains to consider units of orders $10,12,14,15,21,22,33,35,55$ and 77 . We prove that $V(\mathbb{Z} G)$ contains no units of any of these orders, except possibly for order 12 and 24.

Now we consider each case separately.

- Let $u$ be a unit of order 2, 3 or 5 . Using Proposition 2 we immediately obtain that all partial augmentations except one are zero. Thus by Proposition 1 part (ii) of Theorem 1 is proved.

- Let $u$ be a unit of order 4. By (1) and Proposition 2 we get $\nu_{2 a}+\nu_{4 a}+\nu_{4 b}=1$. Now using Proposition 3 we obtain the following system of inequalities:

$$
\begin{aligned}
& \mu_{0}\left(u, \chi_{2}, *\right)=\frac{1}{4}\left(10 \nu_{2 a}+2 \nu_{4 a}+2 \nu_{4 b}+26\right) \geqslant 0 ; \\
& \mu_{2}\left(u, \chi_{2}, *\right)=\frac{1}{4}\left(-10 \nu_{2 a}-2 \nu_{4 a}-2 \nu_{4 b}+26\right) \geqslant 0 ; \\
& \mu_{0}\left(u, \chi_{5}, *\right)=\frac{1}{4}\left(14 \nu_{2 a}+6 \nu_{4 a}-2 \nu_{4 b}+62\right) \geqslant 0 ; \\
& \mu_{2}\left(u, \chi_{5}, *\right)=\frac{1}{4}\left(-14 \nu_{2 a}-6 \nu_{4 a}+2 \nu_{4 b}+62\right) \geqslant 0 .
\end{aligned}
$$

Put $t_{1}=5 \nu_{2 a}+\nu_{4 a}+\nu_{4 b}$ and $t_{2}=7 \nu_{2 a}+3 \nu_{4 a}-\nu_{4 b}$, then $t_{1} \in\{2 r+1 \mid-7 \leqslant r \leqslant 6\}$ and $t_{2} \in\{2 s+1 \mid-16 \leqslant s \leqslant 15\}$. Thus, we obtain the system of linear equations $\nu_{2 a}+\nu_{4 a}+\nu_{4 b}=1, \quad 5 \nu_{2 a}+\nu_{4 a}+\nu_{4 b}=t_{1}, \quad 7 \nu_{2 a}+3 \nu_{4 a}-\nu_{4 b}=t_{2}$. Solving such systems for all possible combinations of values of $t_{1}$ and $t_{2}$, and considering additional inequalities

$$
\begin{aligned}
& \mu_{0}\left(u, \chi_{5}, 3\right)=\frac{1}{4}\left(2 \nu_{2 a}-6 \nu_{4 a}+2 \nu_{4 b}+50\right) \geqslant 0 \\
& \mu_{2}\left(u, \chi_{5}, 3\right)=\frac{1}{4}\left(-2 \nu_{2 a}+6 \nu_{4 a}-2 \nu_{4 b}+50\right) \geqslant 0
\end{aligned}
$$

and also restrictions given by Proposition 5, we get only the 34 integer solutions $\left(\nu_{2 a}, \nu_{4 a}, \nu_{4 b}\right)$ listed in part (iii) of Theorem 1 .

- Let $u$ be a unit of order 6 . By (1) and Proposition 2 we get $\nu_{2 a}+\nu_{3 a}+\nu_{6 a}=1$. 
By Proposition 3 we obtain the following system of inequalities:

$$
\begin{aligned}
& \mu_{1}\left(u, \chi_{2}, *\right)=\frac{1}{6}\left(5 \nu_{2 a}+3 \nu_{3 a}-\nu_{6 a}+13\right) \geqslant 0 ; \\
& \mu_{3}\left(u, \chi_{2}, *\right)=\frac{1}{6}\left(-10 \nu_{2 a}-6 \nu_{3 a}+2 \nu_{6 a}+22\right) \geqslant 0 ; \\
& \mu_{0}\left(u, \chi_{4}, 7\right)=\frac{1}{6}\left(12 \nu_{2 a}+60\right) \geqslant 0 ; \quad \mu_{3}\left(u, \chi_{4}, 7\right)=\frac{1}{6}\left(-12 \nu_{2 a}+48\right) \geqslant 0 ; \\
& \mu_{1}\left(u, \chi_{3}, *\right)=\frac{1}{6}\left(-3 \nu_{2 a}+48\right) \geqslant 0 .
\end{aligned}
$$

Using calculations similar to the previous case, we get only the 15 integer solutions $\left(\nu_{2 a}, \nu_{3 a}, \nu_{6 a}\right)$ listed in part (iv) of Theorem 1.

- Let $u$ be a unit of order 7. By (1) and Proposition 2 we get $\nu_{7 a}+\nu_{7 b}=1$. Using Proposition 3 we obtain the following system of inequalities:

$$
\begin{array}{ll}
\mu_{1}\left(u, \chi_{3}, *\right)=\frac{1}{7}\left(4 \nu_{7 a}-3 \nu_{7 b}+45\right) \geqslant 0 ; & \mu_{3}\left(u, \chi_{3}, *\right)=\frac{1}{7}\left(-3 \nu_{7 a}+4 \nu_{7 b}+45\right) \geqslant 0 ; \\
\mu_{1}\left(u, \chi_{2}, 2\right)=\frac{1}{7}\left(4 \nu_{7 a}-3 \nu_{7 b}+10\right) \geqslant 0 ; & \mu_{3}\left(u, \chi_{2}, 2\right)=\frac{1}{7}\left(-3 \nu_{7 a}+4 \nu_{7 b}+10\right) \geqslant 0 .
\end{array}
$$

Using that $\nu_{7 a}+\nu_{7 b}=1$, we get that $-1 \leqslant \nu_{7 a} \leqslant 2$, and after this it is easy to check that we have only the four integer solutions $\left(\nu_{7 a}, \nu_{7 b}\right)$ listed in part $(\mathrm{v})$ of Theorem 1.

- Let $u$ be a unit of order 11. By (1) and Proposition 2 we get $\nu_{11 a}+\nu_{11 b}=1$. Using Proposition 3 we obtain the following system of inequalities:

$$
\begin{aligned}
\mu_{1}\left(u, \chi_{10}, *\right) & =\frac{1}{11}\left(6 \nu_{11 a}-5 \nu_{11 b}+280\right) \geqslant 0 \\
\mu_{2}\left(u, \chi_{10}, *\right) & =\frac{1}{11}\left(-5 \nu_{11 a}+6 \nu_{11 b}+280\right) \geqslant 0 \\
\mu_{1}\left(u, \chi_{5}, 2\right) & =\frac{1}{11}\left(7 \nu_{11 a}-4 \nu_{11 b}+70\right) \geqslant 0 \\
\mu_{2}\left(u, \chi_{5}, 2\right) & =\frac{1}{11}\left(-4 \nu_{11 a}+7 \nu_{11 b}+70\right) \geqslant 0 \\
\mu_{1}\left(u, \chi_{5}, 3\right) & =\frac{1}{11}\left(6 \nu_{11 a}-5 \nu_{11 b}+49\right) \geqslant 0 ; \\
\mu_{2}\left(u, \chi_{5}, 3\right) & =\frac{1}{11}\left(-5 \nu_{11 a}+6 \nu_{11 b}+49\right) \geqslant 0 .
\end{aligned}
$$

Using calculations similar to the previous case, we get only the ten integer solutions for $\left(\nu_{11 a}, \nu_{11 b}\right)$ listed in part (vi) of Theorem 1.

It remains to prove part (i) of Theorem 1 , considering units of $V(\mathbb{Z} G$ ) of orders $10,14,15,21,22,33,35,55$ and 77 .

- Let $u$ be a unit of order 10. By (1) and Proposition 2 we get $\nu_{2 a}+\nu_{5 a}=1$. Using Proposition 3 we obtain the following system of inequalities:

$$
\begin{aligned}
& \mu_{0}\left(u, \chi_{2}, *\right)=\frac{1}{10}\left(20 \nu_{2 a}+4 \nu_{5 a}+30\right) \geqslant 0 ; \\
& \mu_{5}\left(u, \chi_{2}, *\right)=\frac{1}{10}\left(-20 \nu_{2 a}-4 \nu_{5 a}+20\right) \geqslant 0 ; \\
& \mu_{1}\left(u, \chi_{3}, *\right)=\frac{1}{10}\left(-3 \nu_{2 a}+48\right) \geqslant 0,
\end{aligned}
$$

that has no solutions such that all $\mu_{i}\left(u, \chi_{j}, *\right)$ are non-negative integers.

- Let $u$ be a unit of order 14 . Then by (1) and Proposition 2 we get $\nu_{2 a}+\nu_{7 a}+\nu_{7 b}=1$. We need to consider four cases defined by part (v) of Theorem 1 , but in all of them using Proposition 3 we obtain the same system of inequalities:

$$
\mu_{0}\left(u, \chi_{2}, *\right)=\frac{1}{14}\left(30 \nu_{2 a}+26\right) \geqslant 0 ; \quad \mu_{7}\left(u, \chi_{2}, *\right)=\frac{1}{14}\left(-30 \nu_{2 a}+16\right) \geqslant 0,
$$

which has no solutions such that all $\mu_{i}\left(u, \chi_{j}, *\right)$ are non-negative integers.

- Let $u$ be a unit of order 15 . By (1) and Proposition 2 we get $\nu_{3 a}+\nu_{5 a}=1$. Using 
Proposition 3 we obtain the following system of inequalities:

$$
\begin{aligned}
& \mu_{0}\left(u, \chi_{2}, *\right)=\frac{1}{15}\left(24 \nu_{3 a}+8 \nu_{5 a}+31\right) \geqslant 0 \\
& \mu_{5}\left(u, \chi_{2}, *\right)=\frac{1}{15}\left(-12 \nu_{3 a}-4 \nu_{5 a}+22\right) \geqslant 0 .
\end{aligned}
$$

From this follows that $3 \nu_{3 a}+\nu_{5 a}=-2$, and all conditions together leave us no integer solutions.

- Let $u$ be a unit of order 21. Then by (1) and Proposition 2 we have

$$
\nu_{3 a}+\nu_{7 a}+\nu_{7 b}=1
$$

We need to consider four cases determined by part (v) of Theorem 1. Using Proposition 3 we obtain the following systems of inequalities:

$$
\begin{gathered}
\mu_{0}\left(u, \chi_{2}, *\right)=\frac{1}{21}\left(36 \nu_{3 a}+27\right) \geqslant 0 ; \quad \mu_{7}\left(u, \chi_{2}, *\right)=\frac{1}{21}\left(-18 \nu_{3 a}+18\right) \geqslant 0 ; \\
\mu_{1}\left(u, \chi_{3}, *\right)=\frac{1}{21}\left(3 \nu_{7 a}-4 \nu_{7 b}+\alpha\right) \geqslant 0 ; \quad \mu_{9}\left(u, \chi_{3}, *\right)=\frac{1}{21}\left(-6 \nu_{7 a}+8 \nu_{7 b}+\alpha\right) \geqslant 0 ; \\
\mu_{3}\left(u, \chi_{3}, *\right)=\frac{1}{21}\left(8 \nu_{7 a}-6 \nu_{7 b}+\beta\right) \geqslant 0, \\
\text { where }(\alpha, \beta)=\left\{\begin{array}{lll}
(49,42), & \text { when } \chi\left(u^{3}\right)=\chi(7 a) ; \\
(42,49), & \text { when } & \chi\left(u^{3}\right)=\chi(7 b) ; \\
(56,35), & \text { when } & \chi\left(u^{3}\right)=2 \chi(7 a)-\chi(7 b) ; \\
(35,56), & \text { when } & \chi\left(u^{3}\right)=-\chi(7 a)+2 \chi(7 b),
\end{array}\right.
\end{gathered}
$$

which have no solutions such that all $\mu_{i}\left(u, \chi_{j}, *\right)$ are non-negative integers.

- Let $u$ be a unit of order 22. Then by (1) and Proposition 2 we get

$$
\nu_{2 a}+\nu_{11 a}+\nu_{11 b}=1 \text {. }
$$

We need to consider ten cases determined by part (vi) of Theorem 1 . In each case using Proposition 3 we obtain the following system of inequalities:

$$
\begin{aligned}
\mu_{0}\left(u, \chi_{2}, *\right) & =\frac{1}{22}\left(50 \nu_{2 a}-10 \nu_{11 a}-10 \nu_{11 b}+16\right) \geqslant 0 \\
\mu_{11}\left(u, \chi_{2}, *\right) & =\frac{1}{22}\left(-50 \nu_{2 a}+10 \nu_{11 a}+10 \nu_{11 b}+6\right) \geqslant 0,
\end{aligned}
$$

that has no solutions such that all $\mu_{i}\left(u, \chi_{j}, *\right)$ are non-negative integers.

- Let $u$ be a unit of order 33. Then by (1) and Proposition 2 we get

$$
\nu_{3 a}+\nu_{11 a}+\nu_{11 b}=1 \text {. }
$$

As in the previous case, we need to consider ten cases determined by part (vi) of Theorem 1. In each case using Proposition 3 we obtain the same system:

$$
\begin{aligned}
\mu_{0}\left(u, \chi_{2}, *\right) & =\frac{1}{33}\left(60 \nu_{3 a}-20 \nu_{11 a}-20 \nu_{11 b}+17\right) \geqslant 0 \\
\mu_{11}\left(u, \chi_{2}, *\right) & =\frac{1}{33}\left(-30 \nu_{3 a}+10 \nu_{11 a}+10 \nu_{11 b}+8\right) \geqslant 0,
\end{aligned}
$$

that has no solutions such that all $\mu_{i}\left(u, \chi_{j}, *\right)$ are non-negative integers.

- Let $u$ be a unit of order 35. Then by (1) and Proposition 2 we get $\nu_{5 a}+\nu_{7 a}+\nu_{7 b}=1$. We need to consider four cases defined by part (v) of Theorem 1 , but in all of them using Proposition 3 we obtain the same system of inequalities:

$$
\mu_{0}\left(u, \chi_{2}, *\right)=\frac{1}{35}\left(24 \nu_{5 a}+25\right) \geqslant 0 ; \quad \mu_{0}\left(u, \chi_{7}, 2\right)=\frac{1}{35}\left(-48 \nu_{5 a}+90\right) \geqslant 0,
$$

which has no solutions such that all $\mu_{i}\left(u, \chi_{j}, p\right)$ are non-negative integers.

- Let $u$ be a unit of order 55. Then by (1) and Proposition 2 we get

$$
\nu_{5 a}+\nu_{11 a}+\nu_{11 b}=1 \text {. }
$$


We need to consider ten cases determined by part (vi) of Theorem 1 . In each case using Proposition 3 we obtain the following system of inequalities:

$$
\begin{aligned}
\mu_{0}\left(u, \chi_{2}, *\right) & =\frac{1}{55}\left(40 \nu_{5 a}-40 \nu_{11 a}-40 \nu_{11 b}+15\right) \geqslant 0 ; \\
\mu_{11}\left(u, \chi_{2}, *\right) & =\frac{1}{55}\left(-10 \nu_{5 a}+10 \nu_{11 a}+10 \nu_{11 b}+10\right) \geqslant 0 ; \\
\mu_{1}\left(u, \chi_{10}, *\right) & =\frac{1}{55}\left(-6 \nu_{11 a}+5 \nu_{11 b}+\alpha\right) \geqslant 0 ; \\
\mu_{5}\left(u, \chi_{10}, *\right) & =\frac{1}{55}\left(24 \nu_{11 a}-20 \nu_{11 b}+\alpha\right) \geqslant 0 ; \\
\mu_{1}\left(u, \chi_{2}, *\right) & =\frac{1}{55}\left(\nu_{5 a}-\nu_{11 a}-\nu_{11 b}+21\right) \geqslant 0 \\
\text { where } \quad \alpha & =\left\{\begin{array}{lll}
286, & \text { when } \chi\left(u^{5}\right)=\chi(11 a) ; \\
275, & \text { when } \chi\left(u^{5}\right)=\chi(11 b) ; \\
330, & \text { when } \chi\left(u^{5}\right)=5 \chi(11 a)-4 \chi(11 b) ; \\
253, & \text { when } \chi\left(u^{5}\right)=-2 \chi(11 a)+3 \chi(11 b) ; \\
297, & \text { when } \quad \chi\left(u^{5}\right)=2 \chi(11 a)-\chi(11 b) ; \\
231, & \text { when } \chi\left(u^{5}\right)=-3 \chi(11 a)+4 \chi(11 b) ; \\
308, & \text { when } \quad \chi\left(u^{5}\right)=-4 \chi(11 a)+5 \chi(11 b) ; \\
264, & \text { when } \chi\left(u^{5}\right)=-\chi(11 a)-2 \chi(11 b) ; \\
319, & \text { when } \chi\left(u^{5}\right)=4 \chi(11 a)-3 \chi(11 b) ;
\end{array}\right.
\end{aligned}
$$

that has no solutions such that all $\mu_{i}\left(u, \chi_{j}, *\right)$ are non-negative integers.

- Let $u$ be a unit of order 77. Then by (1) and Proposition 2 we have

$$
\nu_{7 a}+\nu_{7 b}+\nu_{11 a}+\nu_{11 b}=1 \text {. }
$$

We must consider 40 cases determined by parts (v) and (vi) of Theorem 1, but luckily in all of them using Proposition 3 we obtain the same system of inequalities:

$$
\begin{aligned}
\mu_{11}\left(u, \chi_{2}, *\right) & =\frac{1}{77}\left(10 \nu_{11 a}+10 \nu_{11 b}+11\right) \geqslant 0 ; \\
\mu_{0}\left(u, \chi_{2}, *\right) & =\frac{1}{77}\left(-60 \nu_{11 a}-60 \nu_{11 b}+11\right) \geqslant 0,
\end{aligned}
$$

which has no solutions such that all $\mu_{i}\left(u, \chi_{j}, *\right)$ are non-negative integers. This finishes the proof of Theorem 1.

\section{Appendix 1.}

Possible partial augmentations $\left(\nu_{2 a}, \nu_{4 a}, \nu_{4 b}, \nu_{8 a}\right)$ for units of order 8 :

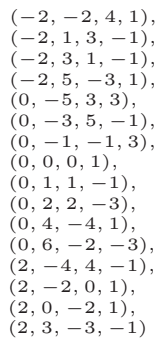

$(-2,-1,3,1)$
$(-2,2,0,1)$
$(-2,3,3,-3)$
$(-2,5,-1,-1)$
$(0,-4,2,3)$
$(0,-2,0,3)$
$(0,-1,1,1)$
$(0,0,2,-1)$
$(0,1,3,-3)$
$(0,3,-5,3)$
$(0,4,-2,-1)$
$(2,-5,1,3)$
$(2,-3,-1,3)$
$(2,-2,2,-1)$
$(2,0,0,-1)$

$(-2,0,2,1)$
$(-2,2,2,-1)$
$(-2,4,-2,1)$
$(-2,5,1,-3)$
$(0,-4,4,1)$
$(0,-2,2,1)$
$(0,-1,3,-1)$
$(0,0,4,-3)$
$(0,2,-4,3)$
$(0,3,-3,1)$
$(0,4,0,-3)$
$(2,-5,3,1)$
$(2,-3,1,1)$
$(2,-1,-3,3)$
$(2,1,-3,1)$
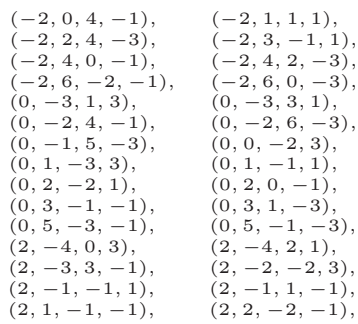

Appendix 2.

Possible partial augmentations $\left(\nu_{2 a}, \nu_{3 a}, \nu_{4 a}, \nu_{4 b}, \nu_{6 a}\right)$ for units of order 12:
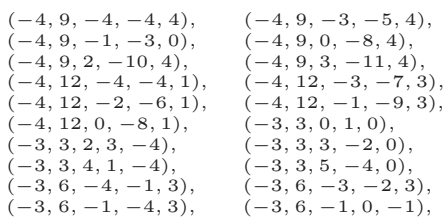

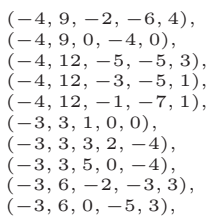

$(-4,9,-1,-7,4)$

$(-4,9,1,-9,4)$,

$(-4,12,-4,-6,3)$,

$(-4,12,-2,-8,3)$

$(-4,12,0,-10,3)$,

$(-3,3,2,-1,0)$

$(-3,3,4,-3,0)$

$(-3,3,6,-5,0)$,

$(-3,6,-2,1,-1)$,
$(-3,6,0,-1,-1)$, 
$(-3,6,1,-6,3)$, $(-3,6,3,-8,3)$, $(-3,6,5,-10,3)$, $(-3,9,-6,-5,6)$, $(-3,9,-5,-2,2)$, $(-3,9,-4,-1,0)$, $(-3,9,-3,-2,0)$, $(-3,9,-2,-3,0)$, $(-3,9,-1,-4,0)$, $(-3,9,0,-5,0)$, $(-3,9,1,-6,0)$ $(-3,9,2,-7,0)$,
$(-3,9,4,-15,6)$, $(-3,9,4,-15,6)$,
$(-3,12,-6,-5,3)$, $(-3,12,-4,-7,3)$, $(-3,12,-2,-9,3)$, $(-3,12,0,-11,3)$, $(-2,0,2,2,-1)$, $(-2,0,4,0,-1)$, $(-2,0,6,-2,-1)$, $(-2,0,8,-4,-1)$, $(-2,3,-2,0,2)$, $(-2,3,-1,3,-2)$, $(-2,3,0,4,-4)$, $(-2,3,1,3,-4)$, $(-2,3,2,2,-4)$, $(-2,3,3,1,-4)$, $(-2,3,4,0,-4)$, $(-2,3,6,-8,2)$, $(-2,3,7,-5,-2)$, $(-2,6,-6,-2,5)$ $(-2,6,-5,1,1)$, $(-2,6,-4,2,-1)$, $(-2,6,-3,1,-1)$,

$(-2,6,-2,0,-1)$ $(-2,6,-1,-1,-1)$, $(-2,6,0,-2,-1)$, $(-2,6,1,-3,-1)$, $(-2,6,2,-4,-1)$, $(-2,6,3,-5,-1)$, $(-2,6,4,-6,-1)$ $(-2,6,6,-10,1)$,
$(-2,9,-8,-6,8)$, $(-2,9,-7,-3,4)$ $(-2,9,-6,-4,4)$ $(-2,9,-5,-5,4)$ $(-2,9,-4,-6,4)$, $(-2,9,-3,-7,4)$, $(-2,9,-2,-8,4)$. $(-2,9,-1,-9,4)$, $(-2,9,0,-10,4)$, $(-2,9,1,-11,4)$, $(-2,9,2,-10,2)$, $(-2,12,-9,-7,7)$, $(-2,12,-7,-9,7)$, $(-2,12,-5,-11,7)$, $(-2,12,-3,-13,7)$, $(-2,12,0,-14,5)$, $(-1,-3,2,9,-6)$, $(-1,-3,4,7,-6)$ $(-1,-3,6,5,-6)$, $(-1,-3,8,3,-6)$, $(-1,-3,10,1,-6)$, $(-1,0,-2,3,1)$, $(-1,0,-1,6,-3)$, $(-1,0,0,7,-5)$, $(-1,0,1,6,-5)$, $(-1,0,2,5,-5)$ $(-1,0,3,4,-5)$, $(-1,0,4,3,-5)$, $(-1,0,5,2,-5)$, $(-1,0,6,1,-5)$, $(-1,0,8,-7,1)$, $(-1,3,-7,2,4)$, $(-1,3,-5,2,2)$, $(-1,3,-4,3,0)$, $(-1,6,-9,-2,7)$, $(-1,6,-7,-4,7)$, $(-1,6,-6,-5,7)$ $(-1,6,-5,-6,7)$ $(-1,6,-4,-7,7)$, $(-1,6,-3,-8,7)$, $(-1,6,-2,-9,7)$, $(-1,6,-1,-10,7)$, $(-1,6,0,-11,7)$, $(-1,6,1,-12,7)$, $(-1,6,2,-11,5)$, $(-1,6,3,-10,3)$, $(-1,9,-10,-7,10)$, $(-1,9,-9,-4,6)$

$(-1,9,-8,-5,6)$,
$(-3,6,1,-2,-1)$, $(-3,6,3,-4,-1)$, $(-3,6,5,-6,-1)$, $(-3,9,-6,-3,4)$, $(-3,9,-4,-7,6)$, $(-3,9,-3,-8,6)$, $(-3,9,-2,-9,6)$, $(-3,9,-1,-10,6)$, $(-3,9,0,-11,6)$ $(-3,9,1,-12,6)$, $(-3,9,2,-13,6)$, $(-3,9,3,-14,6)$, $(-3,12,-7,-6,5)$, $(-3,12,-5,-8,5)$, $(-3,12,-3,-10,5)$, $(-3,12,-1,-12,5)$, $(-3,12,1,-12,3)$, $(-2,0,2,6,-5)$, $(-2,0,4,4,-5)$ $(-2,0,6,2,-5)$, $(-2,0,8,0,-5)$, $(-2,3,-2,4,-2)$, $(-2,3,0,-2,2)$, $(-2,3,1,-3,2)$ $(-2,3,2,-4,2)$ $(-2,3,3,-5,2)$, $(-2,3,4,-6,2)$, $(-2,3,5,-7,2)$, $(-2,3,6,-4,-2)$

$(-2,3,8,-10,2)$, $(-2,6,-6,0,3)$, $(-2,6,-4,-4,5)$, $(-2,6,-3,-5,5)$, $(-2,6,-2,-6,5)$

$(-2,6,-1,-7,5)$

$(-2,6,0,-8,5)$

$(-2,6,1,-9,5)$

$(-2,6,2,-10,5)$,

$(-2,6,3,-11,5)$,

$(-2,6,4,-12,5)$,

$(-2,6,5,-13,5)$,

$(-2,6,6,-8,-1)$

$(-2,9,-8,-4,6)$

$(-2,9,-7,-1,2)$,

$(-2,9,-6,-2,2)$

$(-2,9,-5,-3,2)$

$(-2,9,-4,-4,2)$

$(-2,9,-3,-5,2)$,

$(-2,9,-2,-6,2)$,

$(-2,9,-1,-7,2)$,

$(-2,9,0,-8,2)$

$(-2,9,1,-9,2)$,

$(-2,9,3,-13,4)$,

$(-2,12,-9,-5,5)$,

$(-2,12,-7,-7,5)$,

$(-2,12,-5,-9,5)$,

$(-2,12,-3,-11,5)$

$(-1,-3,0,7,-2)$,
$(-1,-3,3,4,-2)$,

$(-1,-3,5,2,-2)$

$(-1,-3,7,0,-2)$,

$(-1,-3,9,-2,-2)$,

$(-1,-3,11,0,-6)$

$(-1,0,-2,7,-3)$,

$(-1,0,0,1,1)$,

$(-1,0,1,0,1)$,

$(-1,0,2,-1,1)$,

$(-1,0,3,-2,1)$

$(-1,0,4,-3,1)$

$(-1,0,5,-4,1)$

$(-1,0,6,-5,1)$,

$(-1,0,7,-6,1)$,

$(-1,0,8,-3,-3)$

$(-1,3,-6,1,4)$,

$(-1,3,-5,4,0)$,
$(-1,3,-4,5,-2)$,

$(-1,6,-9,0,5)$,

$(-1,6,-7,-2,5)$,

$(-1,6,-6,-3,5)$,

$(-1,6,-5,-4,5)$,

$(-1,6,-4,-5,5)$

$(-1,6,-3,-6,5)$,

$(-1,6,-2,-7,5)$

$(-1,6,-1,-8,5)$

$(-1,6,0,-9,5)$

$(-1,6,1,-10,5)$,

$(-1,6,2,-9,3)$,

$(-1,6,3,-8,1)$

$(-1,9,-10,-5,8)$,

$(-1,9,-9,-2,4)$

$(-1,9,-8,-3,4)$,
$(-3,6,2,-7,3)$, $(-3,6,4,-9,3)$, $(-3,6,6,-11,3)$, $(-3,9,-5,-6,6)$, $(-3,9,-4,-5,4)$, $(-3,9,-3,-6,4)$, $(-3,9,-2,-7,4)$, $(-3,9,-1,-8,4)$, $(-3,9,0,-9,4)$, $(-3,9,1,-10,4)$, $(-3,9,2,-11,4)$, $(-3,9,3,-10,2)$ $(-3,12,-7,-4,3)$, $(-3,12,-5,-6,3)$, $(-3,12,-3,-8,3)$, $(-3,12,-1,-10,3)$, $(-2,0,0,4,-1)$, $(-2,0,3,1,-1)$, $(-2,0,5,-1,-1)$, $(-2,0,7,-3,-1)$, $(-2,3,-4,2,2)$, $(-2,3,-1,-1,2)$, $(-2,3,0,0,0)$, $(-2,3,1,-1,0)$, $(-2,3,2,-2,0)$, $(-2,3,3,-3,0)$, $(-2,3,4,-4,0)$, $(-2,3,5,-3,-2)$, $(-2,3,6,-2,-4)$, $(-2,3,8,-6,-2)$, $(-2,6,-5,-3,5)$, $(-2,6,-4,-2,3)$, $(-2,6,-3,-3,3)$, $(-2,6,-2,-4,3)$, $(-2,6,-1,-5,3)$, $(-2,6,0,-6,3)$, $(-2,6,1,-7,3)$, $(-2,6,2,-8,3)$, $(-2,6,3,-9,3)$, $(-2,6,4,-10,3)$ $(-2,6,5,-9,1)$, $(-2,9,-9,-5,8)$, $(-2,9,-7,-7,8)$, $(-2,9,-6,-8,8)$, $(-2,9,-5,-9,8)$, $(-2,9,-4,-10,8)$ $(-2,9,-3,-11,8)$, $(-2,9,-2,-12,8)$, $(-2,9,-1,-13,8)$. $(-2,9,0,-14,8)$, $(-2,9,1,-15,8)$ $(-2,9,2,-14,6)$, $(-2,9,3,-11,2)$, $(-2,12,-8,-8,7)$, $(-2,12,-6,-10,7)$, $(-2,12,-4,-12,7)$, $(-2,12,-2,-12,5)$, $(-1,-3,1,6,-2)$, $(-1,-3,3,8,-6)$, $(-1,-3,5,6,-6)$, $(-1,-3,7,4,-6)$, $(-1,-3,9,2,-6)$ $(-1,0,-4,5,1)$ $(-1,0,-1,2,1)$, $(-1,0,0,3,-1)$ $(-1,0,1,2,-1)$, $(-1,0,2,1,-1)$ $(-1,0,3,0,-1)$ $(-1,0,4,-1,-1)$, $(-1,0,5,-2,-1)$, $(-1,0,6,-3,-1)$, $(-1,0,7,-2,-3)$ $(-1,0,8,-1,-5)$ $(-1,3,-6,3,2)$, $(-1,3,-4,-1,4)$, $(-1,3,-3,-2,4)$ $(-1,6,-8,-3,7)$, $(-1,6,-7,0,3)$ $(-1,6,-6,-1,3)$, $(-1,6,-5,-2,3)$ $(-1,6,-4,-3,3)$ $(-1,6,-3,-4,3)$, $(-1,6,-2,-5,3)$, $(-1,6,-1,-6,3)$ $(-1,6,0,-7,3)$ $(-1,6,1,-8,3)$ $(-1,6,2,-7,1)$ $(-1,6,4,-9,1)$ $(-1,9,-9,-8,10)$, $(-1,9,-8,-9,10)$, $(-1,9,-7,-10,10)$,

$(-3,6,2,-3,-1)$, $(-3,6,4,-5,-1)$, $(-3,9,-7,-4,6)$, $(-3,9,-5,-4,4)$, $(-3,9,-4,-3,2)$, $(-3,9,-3,-4,2)$, $(-3,9,-2,-5,2)$, $(-3,9,-1,-6,2)$, $(-3,9,0,-7,2)$, $(-3,9,1,-8,2)$, $(-3,9,2,-9,2)$, $(-3,9,3,-8,0)$, $(-3,12,-6,-7,5)$ $(-3,12,-4,-9,5)$ $(-3,12,-2,-11,5)$, $(-3,12,0,-13,5)$, $(-2,0,1,3,-1)$, $(-2,0,3,5,-5)$, $(-2,0,5,3,-5)$, $(-2,0,7,1,-5)$, $(-2,3,-3,1,2)$, $(-2,3,-1,1,0)$, 
$(-1,9,-7,-4,4)$, $(-1,9,-6,-5,4)$, $(-1,9,-5,-6,4)$, $(-1,9,-4,-7,4)$, $(-1,9,-3,-8,4)$, $(-1,9,-1,-10,4)$, $(-1,12,-9,-8,7)$ $(-1,12,-5,-12,7)$, $(0,-9,6,10,-6)$, $(0,-9,10,6,-6)$ $(0,-6,1,9,-3)$, $(0,-6,3,11,-7)$, $(0,-6,3,11,-7)$,
$(0,-6,5,9,-7)$, $(0,-6,5,9,-7)$,
$(0,-6,7,7,-7)$, $(0,-6,9,5,-7)$, $(0,-6,12,2,-7)$, $(0,-3,-2,10,-4)$, $(0,-3,0,4,0)$, $(0,-3,1,3,0)$, $(0,-3,2,2,0)$, $(0,-3,3,1,0)$, $(0,-3,4,0,0)$, $(0,-3,5,-1,0)$, $(0,-3,6,-2,0)$, $(0,-3,7,-3,0)$, $(0,-3,8,-4,0)$, $(0,-3,9,-1,-4)$, $(0,0,-7,5,3)$, $(0,0,-5,5,1)$ $(0,0,-4,6,-1)$, $(0,0,-3,5,-1)$, $(0,0,-2,4,-1)$, $(0,0,-1,3,-1)$, $(0,0,0,2,-1)$, $(0,0,1,1,-1)$, $(0,0,2,0,-1)$, $(0,0,3,-1,-1)$, $(0,0,4,-2,-1)$, $(0,0,5,-3,-1)$, $(0,0,6,-2,-3)$, $(0,3,-9,1,6)$, $(0,3,-7,-1,6)$, $(0,3,-6,-2,6)$, $(0,3,-5,-3,6)$, $(0,3,-4,-4,6)$, $(0,3,-3,-5,6)$, $(0,3,-2,-6,6)$, $(0,3,-1,-7,6)$, $(0,3,0,-8,6)$, $(0,3,1,-9,6)$, $(0,3,2,-8,4)$

$(0,3,3,-7,2)$ $(0,6,-10,-2,7)$, $(0,6,-8,-6,9)$, $(0,6,-7,-7,9)$, $(0,6,-6,-8,9)$ $(0,6,-5,-9,9)$, $(0,6,-4,-10,9)$, $(0,6,-3,-11,9)$, $(0,6,-2,-10,7)$, $(0,6,0,-8,3)$, $(0,9,-8,-6,6)$, $(0,9,-4,-10,6)$ $(1,-9,2,11,-4)$, $(1,-9,5,10,-6)$, $(1,-9,7,8,-6)$, $(1,-9,9,6,-6)$, $(1,-6,-3,10,-1)$, $(1,-6,-1,10,-3)$ $(1,-6,0,11,-5)$, $(1,-6,1,10,-5)$ $(1,-6,2,9,-5)$, $(1,-6,3,8,-5)$ $(1,-6,4,7,-5)$, $(1,-6,5,6,-5)$, $(1,-6,6,5,-5)$, $(1,-6,7,4,-5)$, $(1,-6,8,3,-5)$, $(1,-6,10,1,-5)$, $(1,-3,-6,9,0)$ $(1,-3,-4,5,2)$ $(1,-3,-3,4,2)$ $(1,-3,-2,3,2)$, $(1,-3,-1,2,2)$, $(1,-3,0,1,2)$

$(1,-3,1,0,2)$,

$(1,-3,2,-1,2)$

$(1,-3,3,-2,2)$,

$(1,-3,5,-4,2)$,
$(1,-3,6,-3,0)$
$(-1,9,-6,-11,10)$, $(-1,9,-5,-12,10)$, $(-1,9,-4,-13,10)$, $(-1,9,-3,-14,10)$, $(-1,9,-2,-13,8)$, $(-1,9,0,-11,4)$, $(-1,12,-8,-9,7)$,

$(-1,12,-4,-13,7)$, $(0,-9,7,9,-6)$,
$(0,-9,11,5,-6)$,

$(0,-6,2,8,-3)$,

$(0,-6,4,6,-3)$,

$(0,-6,4,6,-3)$,
$(0,-6,6,4,-3)$,

$(0,-6,6,4,-3)$,
$(0,-6,8,2,-3)$,

$(0,-6,10,0,-3)$

$(0,-3,-4,8,0)$,

$(0,-3,-1,5,0)$

$(0,-3,0,6,-2)$,
$(0,-3,1,5,-2)$,

$(0,-3,2,4,-2)$,

$(0,-3,3,3,-2)$,

$(0,-3,4,2,-2)$,

$(0,-3,5,1,-2)$,

$(0,-3,6,0,-2)$,

$(0,-3,7,-1,-2)$,

$(0,-3,8,-2,-2)$,

$(0,-3,9,1,-6)$,

$(0,0,-6,4,3)$,

$(0,0,-5,7,-1)$,

$(0,0,-4,8,-3)$,

$(0,0,-3,7,-3)$,

$(0,0,-2,6,-3)$,

$(0,0,-1,5,-3)$,

$(0,0,0,4,-3)$,

$(0,0,1,3,-3)$,

$(0,0,2,2,-3)$,

$(0,0,3,1,-3)$,

$(0,0,4,0,-3)$,

$(0,0,5,-1,-3)$,

$(0,0,7,-5,-1)$,

$(0,3,-9,3,4)$,

$(0,3,-7,1,4)$,

$(0,3,-6,0,4)$,

$(0,3,-5,-1,4)$,

$(0,3,-4,-2,4)$,

$(0,3,-3,-3,4)$,

$(0,3,-2,-4,4)$,

$(0,3,-1,-5,4)$,

$(0,3,0,-6,4)$,

$(0,3,1,-7,4)$,

$(0,3,2,-6,2)$,

$(0,3,3,-5,0)$,

$(0,6,-9,-5,9)$,

$(0,6,-8,-4,7)$,

$(0,6,-7,-5,7)$,

$(0,6,-6,-6,7)$,

$(0,6,-5,-7,7)$,

$(0,6,-4,-8,7)$,

$(0,6,-3,-9,7)$,

$(0,6,-2,-6,3)$,

$(0,6,1,-9,3)$,

$(0,9,-7,-7,6)$,

$(0,9,-3,-11,6)$,

$(1,-9,3,10,-4)$,

$(1,-9,6,7,-4)$,

$(1,-9,8,5,-4)$,

$(1,-9,10,3,-4)$,

$(1,-6,-2,9,-1)$,

$(1,-6,-1,12,-5)$,

$(1,-6,0,13,-7)$,

$(1,-6,1,12,-7)$,

$(1,-6,2,11,-7)$,

$(1,-6,3,10,-7)$,

$(1,-6,4,9,-7)$,

$(1,-6,5,8,-7)$

$(1,-6,6,7,-7)$,

$(1,-6,7,6,-7)$,

$(1,-6,8,5,-7)$,

$(1,-6,10,3,-7)$

$(1,-3,-5,6,2)$,

$(1,-3,-4,7,0)$,

$(1,-3,-3,6,0)$,

$(1,-3,-2,5,0)$,

$(1,-3,-1,4,0)$

$(1,-3,0,3,0)$,

$(1,-3,1,2,0)$

$(1,-3,2,1,0)$,

$(1,-3,3,0,0)$,

$(1,-3,4,-1,0)$

$(1,-3,5,-2,0)$,
$(1,-3,6,-1,-2)$,
$(-1,9,-6,-9,8)$,

$(-1,9,-5,-10,8)$,

$(-1,9,-4,-11,8)$,

$(-1,9,-3,-12,8)$,

$(-1,9,-2,-9,4)$,

$(-1,9,1,-12,4)$,

$(-1,12,-7,-10,7)$,

$(-1,12,-3,-14,7)$,

$(0,-9,8,8,-6)$,

$(0,-9,12,4,-6)$,

$(0,-6,2,12,-7)$,

$(0,-6,4,10,-7)$,

$(0,-6,6,8,-7)$

$(0,-6,8,6,-7)$,
$(0,-6,10,4,-7)$,

$(0,-3,-3,7,0)$,

$(0,-3,-1,7,-2)$,

$(0,-3,0,8,-4)$,

$(0,-3,1,7,-4)$,

$(0,-3,2,6,-4)$,

$(0,-3,3,5,-4)$,

$(0,-3,4,4,-4)$,

$(0,-3,5,3,-4)$,

$(0,-3,6,2,-4)$,

$(0,-3,7,1,-4)$,

$(0,-3,8,0,-4)$,

$(0,-3,10,-2,-4)$.

$(0,0,-6,6,1)$,

$(0,0,-4,2,3)$,

$(0,0,-3,1,3)$,

$(0,0,-2,0,3)$,

$(0,0,-1,-1,3)$,

$(0,0,0,-2,3)$,

$(0,0,1,-3,3)$,

$(0,0,2,-4,3)$,

$(0,0,3,-5,3)$,

$(0,0,4,-6,3)$,

$(0,0,5,-7,3)$,

$(0,0,6,-6,1)$,

$(0,0,7,-3,-3)$,

$(0,3,-8,0,6)$,

$(0,3,-7,3,2)$,

$(0,3,-6,2,2)$,

$(0,3,-5,1,2)$,

$(0,3,-4,0,2)$,

$(0,3,-3,-1,2)$,

$(0,3,-2,-2,2)$,

$(0,3,-1,-3,2)$,

$(0,3,0,-4,2)$,

$(0,3,1,-5,2)$,

$(0,3,2,-4,0)$,

$(0,3,4,-6,0)$,

$(0,6,-9,-3,7)$,

$(0,6,-8,-2,5)$,

$(0,6,-7,-3,5)$,

$(0,6,-6,-4,5)$,

$(0,6,-5,-5,5)$

$(0,6,-4,-6,5)$,

$(0,6,-3,-7,5)$,

$(0,6,-1,-11,7)$,

$(0,9,-10,-4,6)$,

$(0,9,-6,-8,6)$,

$(1,-9,0,13,-4)$

$(1,-9,4,9,-4)$,

$(1,-9,6,9,-6)$,

$(1,-9,8,7,-6)$,

$(1,-9,10,5,-6)$,

$(1,-6,-2,13,-5)$

$(1,-6,0,7,-1)$,

$(1,-6,1,6,-1)$,

$(1,-6,2,5,-1)$,

$(1,-6,3,4,-1)$,

$(1,-6,4,3,-1)$,

$(1,-6,5,2,-1)$,

$(1,-6,6,1,-1)$,

$(1,-6,7,0,-1)$,

$(1,-6,8,-1,-1)$,

$(1,-6,9,2,-5)$,

$(1,-3,-7,8,2)$,

$(1,-3,-5,8,0)$,
$(1,-3,-4,9,-2)$,

$(1,-3,-3,8,-2)$,

$(1,-3,-2,7,-2)$,

$(1,-3,-1,6,-2)$,

$(1,-3,0,5,-2)$,

$(1,-3,1,4,-2)$,

$(1,-3,2,3,-2)$,

$(1,-3,3,2,-2)$,

$(1,-3,4,1,-2)$,

$(1,-3,5,0,-2)$,
$(1,-3,6,1,-4)$, 
Torsion units
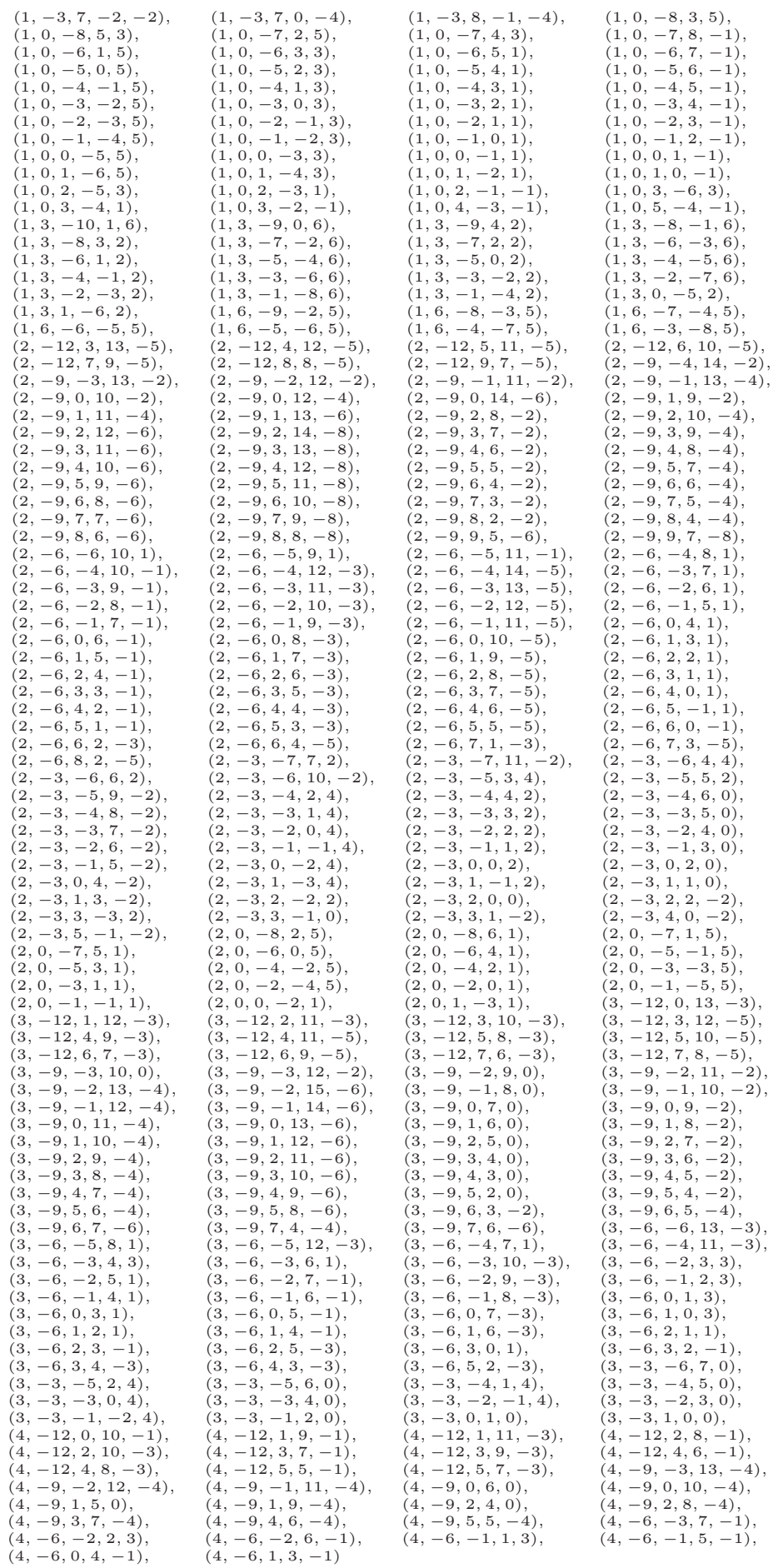

https://doi.org/10.1112/S1461157000000516 Published online by Caßg1pridge University Press 


\section{References}

1. V. A. Artamonov and A. A. Bovdi, 'Integral group rings: groups of invertible elements and classical K-theory', Algebra. Topology. Geometry (Russian), Itogi Nauki i Tekhniki 27 (Akad. Nauk SSSR, Vsesoyuz. Inst. Nauchn. i Tekhn. Inform., Moscow, 1989) 3-43, 232. 28

2. S. D. Berman, 'On the equation $x^{m}=1$ in an integral group ring', Ukrain. Mat. Ž. 7 (1955) 253-261. 29

3. F. M. Bleher and W. KimmerLe, 'On the structure of integral group rings of sporadic groups', LMS J. Comput. Math. 3 (2000) 274-306 (electronic). 28

4. V. Bovdi and M. Hertweck, 'Zassenhaus conjecture for central extensions of $S_{5}$ ', J. Group Theory, to appear, arXiv:math.RA/0609435v1. 28, 30

5. V. Bovdi, C. Höfert and W. Kimmerle, 'On the first Zassenhaus conjecture for integral group rings', Publ. Math. Debrecen 65 (2004) 291-303. 28

6. V. Bovdi, E. Jespers and A. Konovalov, 'Torsion units in integral group rings of Janko simple groups', Preprint, 2006, arXiv:math/0608441v3. 28

7. V. Bovd and A. Konovalov, 'Integral group ring of the first Mathieu simple group', Groups St. Andrews 2005, Vol. I, London Math. Soc. Lecture Note Ser. 339 (Cambridge University Press, Cambridge, 2007) 237-245. 28

8. V. Bovdi and A. Konovalov, 'Integral group ring of the Mathieu simple group $M_{23}$ ', Comm. Algebra, to appear, arXiv:math/0612640v2. 28

9. V. Bovdi, A. Konovalov, R. Rossmanith and Cs. Schneider, LAGUNA - Lie AlGebras and UNits of group Algebras, version 3.4, 2007, http://ukrgap.exponenta.ru/laguna.htm. 30

10. V. Bovdi, A. Konovalov and S. Siciliano, 'Integral group ring of the Mathieu simple group $M_{12}$ ', Rend. Circ. Mat. Palermo (2) 56 (2007) 125136. 28

11. J.A. Cohn and D. Livingstone, 'On the structure of group algebras. I', Canad. J. Math. 17 (1965) 583-593. 30, 31

12. J. H. Conway, R. T. Curtis, S. P. Norton, R. A. Parker and R. A. Wilson, Atlas of Finite Groups. Maximal subgroups and ordinary characters for simple groups. With computational assistance from J. G. Thackray (Oxford University Press, Eynsham, 1985). 31

13. The GAP Group, GAP - Groups, Algorithms, and Programming, Version 4.4.9, 2006, http://www.gap-system.org. 29, 31

14. M. HERTWECK, 'On the torsion units of some integral group rings', Algebra Colloq. 13 (2006) 329-348. 28, 30

15. M. HERTWECK, 'Partial augmentations and Brauer character values of torsion units in group rings', Comm. Algebra, to appear, arXiv:math.RA/0612429v2. 28,30

16. M. HERTWECK, 'Torsion units in integral group rings of certain metabelian groups', Proc. Edinb. Math. Soc., to appear. 28 
17. C. Höfert and W. Kimmerle, 'On torsion units of integral group rings of groups of small order', Groups, rings and group rings, Lect. Notes Pure Appl. Math. 248 (Chapman \& Hall/CRC, Boca Raton, FL, 2006) 243-252. 28

18. C. Jansen, K. Lux, R. Parker and R. Willson, An Atlas of Brauer Characters, London Mathematical Society Monographs New Series 11 (Clarendon Press, Oxford University Press, New York, 1995), Appendix 2 by T. Breuer and S. Norton. 31

19. W. Kimmerle, 'On the prime graph of the unit group of integral group rings of finite groups', Groups, rings and algebras, Contemp. Math. 420 (American Mathematical Society, Providence, RI, 2006) 215-228. 28

20. I. S. Luthar and I. B. S. PAssi, 'Zassenhaus conjecture for $A_{5}$ ', Proc. Indian Acad. Sci. Math. Sci. 99 (1989) 1-5. 28, 30

21. I. S. Luthar and P. Trama, 'Zassenhaus conjecture for $S_{5}$ ', Comm. Algebra 19 (1991) 2353-2362. 28

22. Z. Marciniak, J. Ritter, S. K. Sehgal and A. Weiss, 'Torsion units in integral group rings of some metabelian groups. II', J. Number Theory 25 (1987) 340-352. 30

23. R. SAndLing, 'Graham Higman's thesis "Units in group rings"', Integral representations and applications, Oberwolfach, 1980, Lecture Notes in Mathematics 882 (Springer, Berlin, 1981) 93-116. 29

24. E. Wiтt, 'Die 5-fach transitiven Gruppen von Mathieu', Abh. Math. Semin. Hansische Univ. 12 (1938) 256-264. 29

25. H. Zassenhaus, 'On the torsion units of finite group rings' (Portuguese), Studies in mathematics (in honor of A. Almeida Costa) (Instituto de Alta Cultura, Lisbon, 1974) 119-126. 28

\section{A. Bovdi vbovdi@math.klte.hu}

Institute of Mathematics, University of Debrecen P.O. Box 12, H-4010 Debrecen, Hungary Institute of Mathematics and Informatics, College of Nyíregyháza Sóstói út 31/b, H-4410 Nyíregyháza, Hungary

A. B. Konovalov konovalov@member.ams.org

S. Linton sal@cs.st-and.ac.uk

School of Computer Science, University of St Andrews

North Haugh, St Andrews, Fife, KY16 9SX, Scotland 